\title{
Tailoring the emissive properties of photocathodes through materials engineering: ultra- thin multilayers
}

\author{
Daniel Velázquez, Rachel Seibert, Hasitha Ganegoda, Daniel Olive, ${ }^{1,2}$ Amy Rice, Kevin Logan, \\ Zikri Yusof, Linda Spentzouris and Jeff Terry*
}

Physics Department, Illinois Institute of Technology, Chicago, Illinois 60616, USA

(*) Corresponding author

${ }^{1}$ Chemical Sciences Division, Lawrence Berkeley National Laboratory, Berkeley, CA 94720, USA

${ }^{2}$ Materials Science and Technology Division, Los Alamos National Laboratory, Los Alamos, New Mexico, 87545, USA 
Abstract

We report on an experimental verification that emission properties of photocathodes can be manipulated through the engineering of the surface electronic structure. Ultrathin multilayered $\mathrm{MgO} / \mathrm{Ag}(001) / \mathrm{MgO}$ films were grown by pulsed laser deposition, tuning the thickness $n$ of the flanking $\mathrm{MgO}$ layers to $0,2,3$, and 4 monolayers. We observed an increase in quantum efficiency and simultaneous decrease in work function with layer thickness. The scale and trend direction of measurements are in good but not excellent agreement with theory. Angle resolved photoemission data for the multilayered sample $n=3$ showed that the emission profile has a metallic-like momentum dispersion. Deviations from theoretical predictions [K. Németh et al., PRL 104, 046801 (2010)] are attributed to imperfections of real surfaces in contrast with the ideal surfaces of the calculation. Photoemissive properties of cathodes are critical for electron beam applications such as photoinjectors for Free Electron Lasers (FEL) and Energy Recovery Linacs (ERL). An ideal photoemitter has a high quantum efficiency, low work function, low intrinsic emittance and long lifetime. It has been demonstrated here that emission properties may be systematically tailored by control of layer thickness in ultrathin multilayered structures. The reproducibility of the emission parameters under specific growth conditions is excellent, even though the interfaces themselves have varying degrees of roughness. 


\section{Keywords}

Pulsed laser deposition, RHEED, epitaxy, photocathode, work function, quantum efficiency
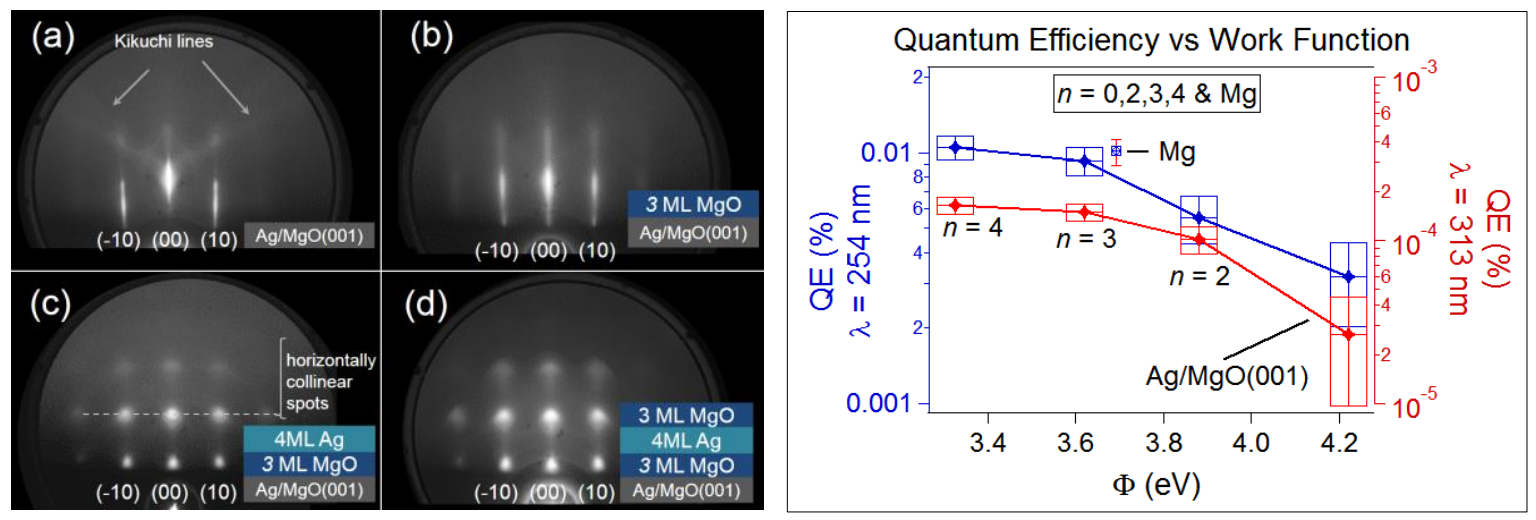

Graphical Abstract 


\section{Introduction}

Much of the development of photocathode materials has been aimed at the growth of photoemissive thin films with low work function (WF), and high quantum efficiency (QE) [1]. It has been shown, both theoretically and experimentally, that metal-insulator junctions can lead to the modification of the WF for coverages of a few monolayers (ML) of metal oxides on metallic substrates [2-6]. Reduction of WF and increase of QE can be achieved simultaneously by coating metal surfaces with $\mathrm{Cs}, \mathrm{CsBr}$, Y and $\mathrm{Mg}$ [7-11]. Cs ion implantation on $\mathrm{Cu}, \mathrm{Ag}$, and $\mathrm{Au}$ has been shown to reduce the WF and increase QE while still retaining the robustness of the metal; nonetheless, the crystalline quality of the substrate is sacrificed [12]. Since low intrinsic emittance beams play an increasingly important role for some photoinjector driven applications, maintaining a high quality surface can be advantageous in avoiding emittance degradation [13].

A theoretical model by Németh, et al. [2] describes the density functional theory (DFT) simulation of a multilayered structure $\mathrm{MgO} / \mathrm{Ag}(001) / \mathrm{MgO}$ in the configuration of $4 \mathrm{ML}$ of $\mathrm{Ag}(001)$ flanked by $n \mathrm{ML}$ of $\mathrm{MgO}$. This model indicates that 2 or $3 \mathrm{ML}$ of $\mathrm{MgO}$ can reduce the emittance of an electron beam, because the surface band structure exhibits a narrowing of the range of surface-parallel momenta near the $\Gamma$-point neighboring the Fermi level. Since the surface-parallel momenta of the electrons are conserved in prompt emission, the angular spread of emitted electrons (and so the intrinsic beam emittance) is reduced. In addition, this and another similar model [5] predict a WF drop of 1-1.5 eV from that of $4 \mathrm{ML}$ of $\mathrm{Ag}(001)$. Measurements using atomic and Kelvin probe force microscopies (AFM and KPFM) show that even a one layer deposition of $\mathrm{MgO}$ on a $\mathrm{Ag}(001)$ single crystal substrate produces a WF drop of $1.1 \mathrm{eV}$ (1 ML) and $1.4 \mathrm{eV}$ (2 ML) from that of the bare substrate $[3,4]$. Recently, an effort to quantify the effect on emittance of a single $4 \mathrm{ML}$ coating of $\mathrm{MgO}$ on $\mathrm{Ag}(001)$ was carried out using Angle Resolved Photoelectron Spectroscopy (ARPES) by Droubay, et al. [14]. The photon energy was not optimized, so the emittance of the coated sample was larger than from the substrate alone. However, an enhancement of the photoemission intensity at the $\Gamma$-point near the Fermi level was observed for the coated versus the uncoated $\mathrm{Ag}(001)$ substrate.

Here the dependence of emission behavior on the thickness of the $\mathrm{MgO}$ layers of multilayered $\mathrm{MgO} / \mathrm{Ag} / \mathrm{MgO}$ structures was examined. The high quality multilayer films were fabricated using pulsed laser deposition. Reflection High-Energy Electron Diffraction (RHEED) [15-17] and Photoelectron Spectroscopy (PES) [18,19] were used to monitor the formation of the highly oriented, crystalline, multilayer films, and verify the chemical structure of each. The Kelvin Probe technique was used to determine any changes in the work function as a function of the thickness of the MgO layers. QE was measured in vacuum by grounding the samples, illuminating them with UV light and collecting the photocurrent using an anode at $450 \mathrm{~V}$. ARPES was used to measure the angular emission profile of the multilayer $n=3$.

\section{Materials and Methods}

Pulsed Laser Deposition (PLD), a state-of-the-art ultra-high vacuum film synthesis technique [20-23], was used for growth of all films described here. The laser fluence for all instances was $5-6 \mathrm{~J} / \mathrm{cm}^{2}$. Substrate heating was performed using a continuous wave $\mathrm{CO}_{2}$ laser $(10.6 \mu \mathrm{m})$ that impinges on the back of the substrate through a zinc-selenide window. Film growth monitoring was carried out using in-situ RHEED. The deposition rate was calibrated before growth of each film using a quartz crystal microbalance (QCM) with an uncertainty of less than $10 \%$ of the film thickness. Thickness measurements were independently verified using ellipsometry showing a small average discrepancy of $\pm 0.03 \AA$. It was found in a separate study that the roughness of the deposited surfaces was reproducibly dependent on the substrate temperature during deposition. The surfaces of the films for different layer thicknesses were consistent in quality from sample to sample.

\section{Results and Discussion}

\subsection{Synthesis and Structural Characterization}

The multilayer $\mathrm{MgO} / \mathrm{Ag} / \mathrm{MgO}$ structures, in the (001) orientation, were fabricated on smooth epitaxial $40 \mathrm{~nm}$ Ag buffer films deposited on $\mathrm{MgO}(001)$ in order to substitute more expensive commercially available single crystal metallic substrates. Multilayers consisted of a $4 \mathrm{ML} \mathrm{Ag}$ interlayer flanked by $n=2,3,4 \mathrm{MgO}$ monolayers, where the nominal monolayer thicknesses (i.e. half of the lattice parameter) are: $2.106 \AA$ and $2.045 \AA$ for $(001) \mathrm{MgO}$ and Ag, respectively [2]. The deposition temperature was $130{ }^{\circ} \mathrm{C}$ and the chamber base pressure was $\sim 5 \times 10^{-9}$ Torr for all films.

After growth, the films were initially characterized in order to study their structural properties. During growth the films were monitored in situ using RHEED in order to examine their crystalline structure. An example of multilayer evolution (for $n=3 \mathrm{ML})$ during growth, examined by RHEED, is shown in Figure 1. Figure 1 (a) shows the pattern of the $\mathrm{Ag} / \mathrm{MgO}(001)$ growth platform. Thin rods and Kikuchi lines as well as the absence of transmission-like features indicate that the film has become a terraced 2-dimensional surface $[6,15]$. The RHEED system was calibrated using the pattern from $\mathrm{MgO}(001)($ not 
shown). The calibration showed that the lattice constant for the $\mathrm{Ag} / \mathrm{MgO}(001)$ pattern was $4.01 \pm 0.03 \AA, \sim 2 \%$ smaller than the nominal value of $4.09 \AA$ for bulk $\operatorname{Ag}(001)$ [2]. Figure 1 (b)-(d) show RHEED patterns of the sequential deposition of $\mathrm{MgO}$ (3 ML), $\mathrm{Ag}$ (4 ML), $\mathrm{MgO}(3 \mathrm{ML})$ on $\mathrm{Ag} / \mathrm{MgO}(001)$. Epitaxial growth is manifested by the absence of polycrystalline features (rings and/or curved rods) [15-17]. The appearance of distinctive horizontally collinear features is indicative of transmission diffraction from 3D surface features [17], thus it is clear that the films grew in the form of highly ordered epitaxial islands. All patterns were taken along the [100] azimuth of $\mathrm{MgO}(001)$.

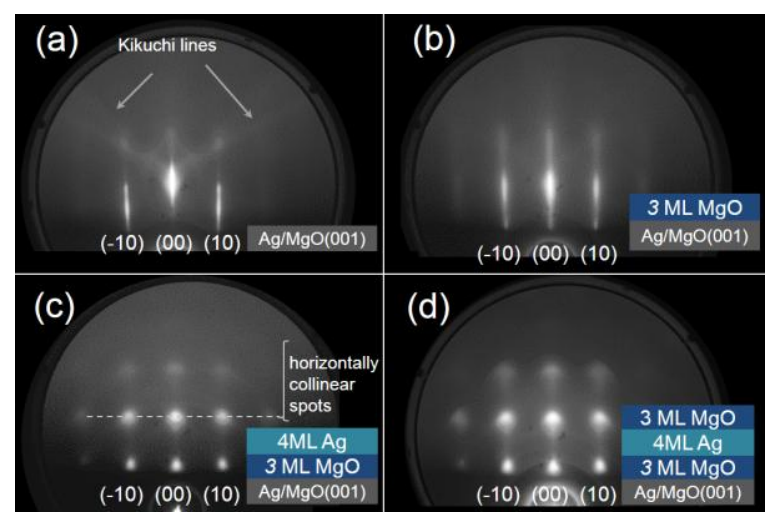

Figure 1. RHEED patterns during deposition of multilayer $n=3 \mathrm{ML}$ : (a) $\mathrm{Ag} / \mathrm{MgO}(001)$ support, (b)-(d) multilayer progression.

The multilayer surfaces were then imaged by STM in order to confirm the results from RHEED. Figure 2 depicts the STM images corresponding to the multilayer $n=3 \mathrm{ML}$ described in Figure 1. Figure 2 (a) confirms the highly ordered, terraced surface finish of the $\mathrm{Ag} / \mathrm{MgO}(001)$ growth platform, and Figure 2 (b)-(d) shows the formation of the multilayer as epitaxial nano-islands. The average and rms surface roughness for the $\mathrm{Ag} / \mathrm{MgO}(001)$ support were measured to be $0.42 \pm 0.03 \mathrm{~nm}$ and $0.52 \pm 0.04 \mathrm{~nm}$, respectively. The average and rms surface roughness for the multilayer surface $n=3 \mathrm{ML}$ were measured to be $0.56 \pm 0.03 \mathrm{~nm}$ and $0.71 \pm 0.03 \mathrm{~nm}$, respectively. Roughness was sampled over $100 \times 100 \mathrm{~nm}^{2}$ areas.

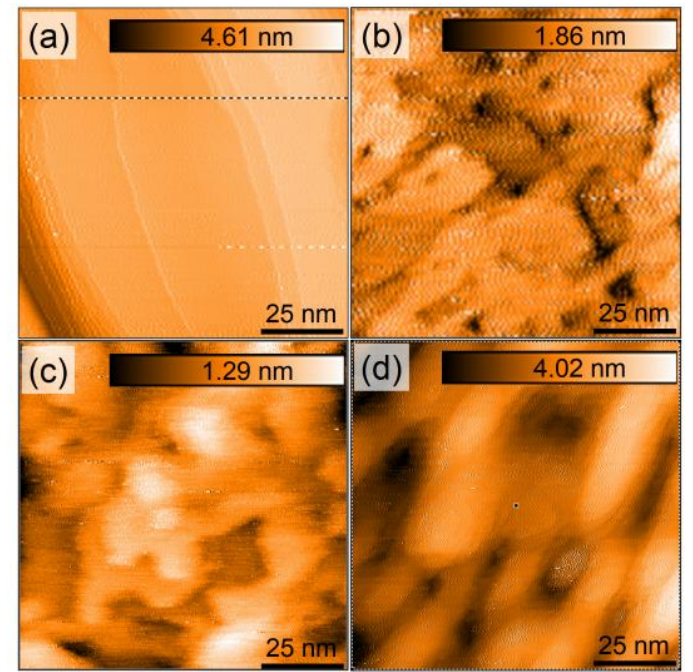

Figure 2. Ex-situ STM at different stages of growth of multilayer $n=3 \mathrm{ML}$ as shown in Figure 1: (a) $\mathrm{Ag} / \mathrm{MgO}(001)$, (b)-(d) multilayer progression.

In addition to the characterization of the physical structure by RHEED and STM, ex-situ PES was used to track the chemical structure of the multilayered films at different stages of deposition. Figure 3 shows Al $\mathrm{K}_{\alpha}$ PES spectra of the evolution of deposition for the $n=3 \mathrm{ML}$ example in Figures 1 and 2; starting from the $\mathrm{Ag} / \mathrm{MgO}(001)$ single crystal surface through the sequence $\mathrm{MgO}(n=3 \mathrm{ML}), \mathrm{Ag}(4 \mathrm{ML}), \mathrm{MgO}(n=3 \mathrm{ML})$. The stages of deposition can be observed in the modulation of the $\mathrm{Mg} 2 \mathrm{~s}$ and $2 \mathrm{p}$ peaks as their intensities increase and decrease according to the surface finish at any given step. A small shift of 0.4-0.6 eV toward higher kinetic energy (lower binding energy) was observed in the $\mathrm{Mg}$ core levels. 
This is likely the result of the electron density displacement from $\mathrm{Mg}-\mathrm{O}$ bonds due to the formation of interfacial states with Ag-O bonds. RHEED patterns, STM images and XPS spectra collected for multilayers $n=2 \mathrm{ML}$ and 4 ML showed similar results and trends, which indicate the growth of epitaxial and stoichiometric films with surface roughness less than $1 \mathrm{~nm}$ over sampled areas of $100 \times 100 \mathrm{~nm}^{2}$.

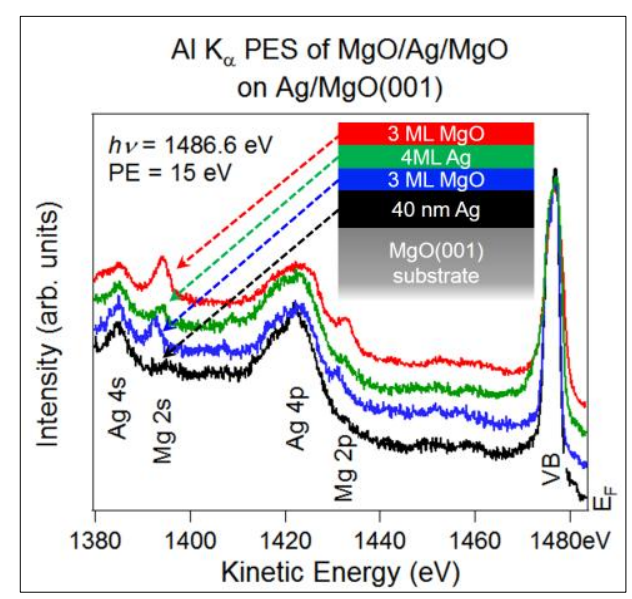

Figure 3. PES spectra showing the stages of deposition of $\mathrm{MgO} / \mathrm{Ag} / \mathrm{MgO}$ on $\mathrm{Ag} / \mathrm{MgO}(001)$. The formation of the multilayer is evidenced by the increase (decrease) of intensity of the $\mathrm{Mg}(\mathrm{Ag})$ peaks.

\subsection{Photoemissive Properties}

In order to test the theoretical model by Nemeth et al. [2], the films were characterized as photoemitters by measuring their WF and QE. The theoretical model was reproduced in order to verify that the calculation was not dependent on the particular model used. In addition, an initial measurement of angular emission is provided for the multilayer with $n=3 \mathrm{ML}$.

Relative work function measurements of multilayers $n=2,3$ and 4 ML were made using the Kelvin Probe technique [24]. The probe tip WF was calibrated using commercially available HOPG and high purity (99.97\%) polycrystalline $\mathrm{Nb}$ foil. Figure 4 shows the relative WF, $\Delta \Phi$, of multilayers with respect to $\mathrm{Ag} / \mathrm{MgO}(001)$ as a function of multilayer thickness $(n)$. A monotonic decrease in the WF with multilayered film thickness (given as the number of $\mathrm{MgO}$ monolayers) was observed. For comparison, Density Functional Theory (DFT) calculations are plotted as well.

The WF for multilayers $n=2,3$ and 4 ML was calculated using the software QuantumWise Atomistic Toolkit with Virtual NanoLab, which uses the formalisms of linear combination of atomic orbitals (LCAO) and the generalized gradient approximation (GGA) [25]. We used the PW91 functional with cutoff at $1360 \mathrm{eV}$ for bulk and $1000 \mathrm{eV}$ for slabs. The atomic grids were $23 \times 23 \times 23$ (bulk), $16 \times 16 \times 1$ (slabs). Geometric optimization yielded maximum stress values of $0.001 \mathrm{eV} / \AA$ and lattice vectors within $4 \%$ of their nominal value, similar to previous results [2]. Our DFT calculations and those from reference [2] for these films suggest an initial sharp drop in WF of close to $1 \mathrm{eV}$ for the multilayer system with $n=1 \mathrm{ML}$ of $\mathrm{MgO}$; this reduction is almost the same for 2, 3 and $4 \mathrm{MgO} \mathrm{ML}$. The measurements presented here show a somewhat linear decrease $\Delta \Phi$ of $-0.34 \mathrm{eV},-0.60 \mathrm{eV}$ and $-0.89 \mathrm{eV}( \pm 0.07 \mathrm{eV})$ for $n=2,3$ and $4 \mathrm{ML}$, respectively. As suggested by the RHEED patterns (Figure 3), the multilayers must have a roughness, consisting of islands of material of varying height and perhaps, finish ( $\mathrm{Ag}$ or $\mathrm{MgO})$. It is suggestive that the monotone WF drop is correlated with the surface coverage, which is likely due to the average contact potential difference (CPD) measurement under the macroscopic $2 \mathrm{~mm}$ diameter tip. The effect of roughness thus results in the unexpected benefit of providing the means for finer control of the WF change with multilayer thickness than predicted by DFT calculations of perfect surfaces. We expect that a more realistic theoretical description of the multilayers will require the inclusion of interface roughness. 


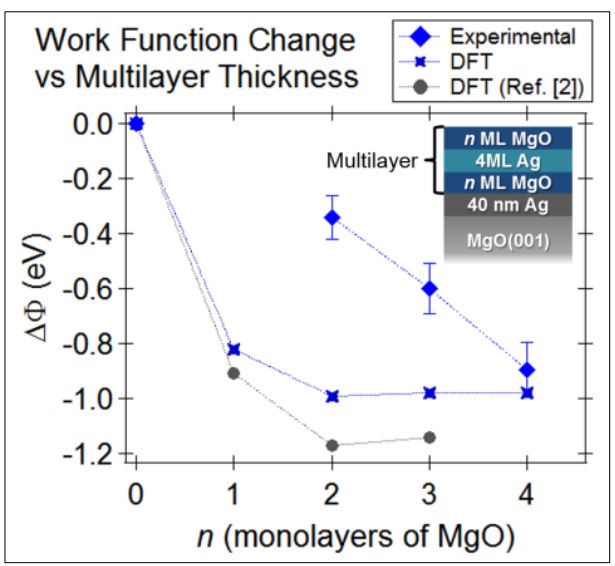

Figure 4. WF measurements of multilayers $n=2,3,4$ for the orientations compared to our DFT calculations and those from [2].

The reduction in the WF suggests an increase in QE for a fixed photoexcitation energy, as deeper binding energy states can be probed. Figure 5 shows measurements of QE versus WF for $\operatorname{Ag}(001)$, multilayer $(n=2,3,4 \mathrm{ML})$ and polycrystalline $\mathrm{Mg}$ surfaces. The measurements were conducted at $25^{\circ} \mathrm{C}$ in a vacuum environment at $\sim 10^{-4}$ Torr using a white light source (Hgarc lamp) with filters to select $254 \mathrm{~nm}$ and $313 \mathrm{~nm}$ wavelengths. The samples were grounded and illuminated while the photocurrent was collected by an electrode at a voltage of $450 \mathrm{~V}$ using a pico-ammeter. The background signal was recorded to be 1-2 orders of magnitude weaker than the photocathode current. In order to compare the photoemissive performance of the multilayered films, the WF and QE of Mg (a high QE alkaline earth metal routinely used as a photocathode coating and even in bulk as a photocathode itself [26-29]), were measured as well. The QE scales in the plots were adjusted to align the values of $\mathrm{Mg}$ for $254 \mathrm{~nm}$ and $313 \mathrm{~nm}$ wavelengths in order to facilitate visual comparison between the QE of thin films and $\mathrm{Mg}$. The increase in the QE of the multilayers is attributed to the reduction in WF with film thickness. For both wavelengths used, the increase in QE ranged from about half to one order of magnitude. At $313 \mathrm{~nm}$ the rise in QE was not enough to reach the level of $\mathrm{Mg}$. However at $254 \mathrm{~nm}$, the increase in QE for multilayers $n=3$ and $4 \mathrm{ML}$ was more substantial, reaching the level of $\mathrm{Mg}$ within error.

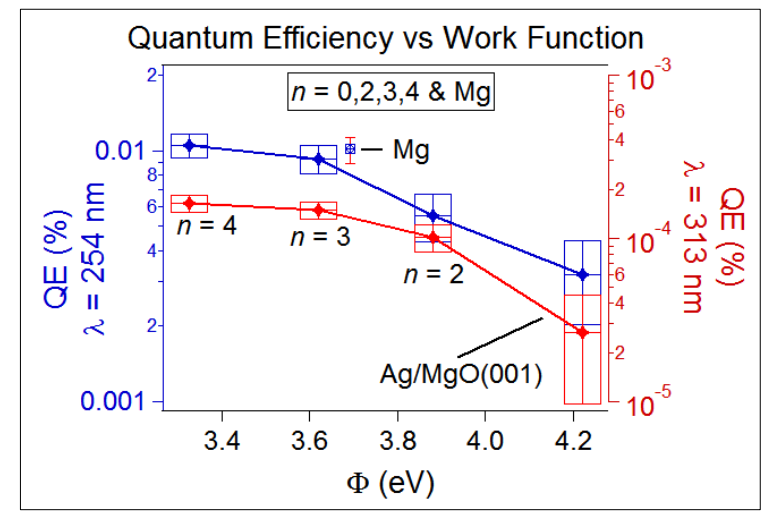

Figure 5. Plots of QE vs WF of $\mathrm{Mg}, \mathrm{Ag} / \mathrm{MgO}(001)$ and multilayers $n=2,3,4 \mathrm{ML}$ using $254 \mathrm{~nm}$ and $313 \mathrm{~nm}$ wavelengths.

We measured the ARPES spectrum of a multilayer as an initial attempt to determine the angular emission profile of the multilayers. The spectrum of the multilayer $n=3 \mathrm{ML}$ was obtained using synchrotron radiation with photon energy of $22 \mathrm{eV}$ at pass energy of $5 \mathrm{eV}$ at the U13UB beamline from NSLS at BNL. The Fermi level was measured at $17.4 \mathrm{eV}$ using a metallic $\mathrm{Ag}(001)$ surface for calibration. The samples were aligned at fixed azimuthal orientation in order to collect energy distribution curves (EDCs) at various positions of the polar angle $(\theta)$ ranging from $0^{\circ}$ to $45^{\circ}$. The low-binding-energy region $(0-2 \mathrm{eV})$ was modeled with Gaussian fits of $0.85 \mathrm{eV} \mathrm{FWHM} \mathrm{for} \mathrm{each} \mathrm{EDC.} \mathrm{The} \mathrm{background} \mathrm{was} \mathrm{modeled} \mathrm{as} \mathrm{an} \mathrm{exponential}$ decaying curve. Due to its complex structure, the density of states of the $\mathrm{Ag} 4 \mathrm{~d}$ core level (roughly $4.5 \mathrm{eV}$ to $8.5 \mathrm{eV}$ ), was approximated with a 4-Gaussian envelope. Figure 6 shows the momentum-transformed dispersion of the peak-fit positions within the first $2 \mathrm{eV}$. The dispersion observed resembles that of narrow metallic surface bands due to the average over relatively ordered islands and grains, as seen in Figures 1 and 2. This behavior has been previously observed for surfaces with 
similar physical structure [14,30,31]. We expect these ARPES spectra to be consistent given the reproducibility of the various surfaces studied here.

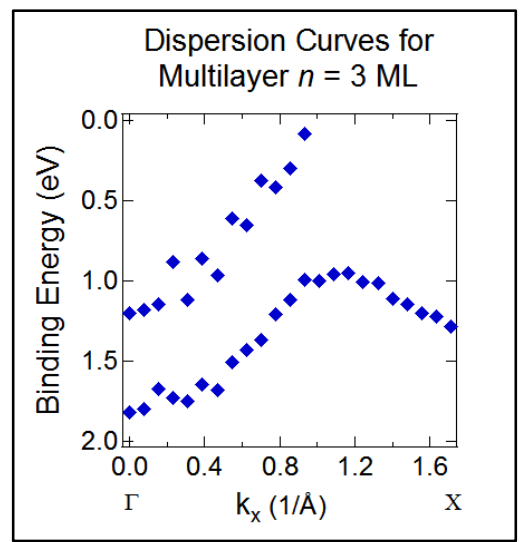

Figure 6. Positions of peak-fits from ARPES spectra near the Fermi level of the multilayer $n=3 \mathrm{ML}$. This is approximately an average over the band structure of the islands on the surface.

\section{Conclusion}

To conclude, we have provided evidence showing one can control the photoemissive properties of photoemitters by engineering their physical and hence, electronic structure. It was shown that simultaneous and gradual decreases in WF and increases in $\mathrm{QE}$ are dependent on the thickness of $\mathrm{MgO} / \mathrm{Ag}(001) / \mathrm{MgO}$ multilayers. Using UV-ARPES we have shown that the angular profile of emission of the $n=3 \mathrm{ML}$ multilayered exhibits a metallic-like dispersion. Demonstration of the ability to tailor emission properties via the precision deposition of thin crystalline layers is an encouraging result that supports efforts to fabricate designer photocathodes for increased performance in photoinjector applications.

\section{Acknowledgements}

The authors thank the staff of AWA at ANL, Eric Wisniewski, Katherine Harkay and Károly Németh for valuable discussions and the facilitation of resources crucial for the completion of these studies. We also thank the staff of CMP and U13UB beamline from NSLS at BNL. This work was funded by the National Science Foundation under the grant no. 0969989, the Department of Energy under the grant no. DE-SC0007952 and the U.S. Department of Education through the GAANN Fellowship Program.

\section{References}

[1] D. H. Dowell et al., Nucl. Instrum. Meth. A 622, 3 (2010).

[2] K. Nemeth et al., Phys. Rev. Lett. 104, 046801 (2010).

[3] M. Bieletzki et al., Phys. Chem. Chem. Phys. 12 (2010).

[4] T. König et al., J. Phys. Chem. C 113 (2009).

[5] L. Giordano et al., Phys. Rev. B 73, 045414 (2005).

[6] D. Velazquez et al., in International Particle Accelerator Conference, Richmond, VA, USA (2015).

[7] Z. Liu et al., Appl. Phys. Lett. 90, 231115 (2007).

[8] R. A. Powell et al., Phys. Rev. B 8, 3987 (1973).

[9] A. Lorusso et al., Appl. Surf. Sci. 258, 22 (2012)

[10] P. Miglietta et al., Appl. Surf. Sci. 255, 20 (2012)

[11] L. Cultrera et al., Appl. Surf. Sci. 253, 15 (2007)

[12] K. Zhao et al., Nucl. Instrum. Meth. A 445 (2000).

[13] Z. Zhang et al., Phys. Rev. ST Accel. Beams 18, 053401 (2015).

[14] T. Droubay et al., Phys. Rev. Lett. 112, 067601 (2014).

[15] A. Ichimiya and P. I. Cohen, Reflection High Energy Electron Diffraction, (Cambridge University Press, New York, 2004).

[16] W. Braun, Applied RHEED: Reflection High-energy Electron Diffraction During Crystal Growth (Springer-Verlag, Heidelberg, 1999).

[17] G.-C. Wang and T.-M. Lu, RHEED Transmission Mode and RHEED Pole Figure," in RHEED Transmission Mode and Pole Figures: Thin Film and Nanostructure Texture Analysis (Springer, New York, 2013).

[18] S. Hüfner, Photoelectron Spectroscopy: Principles and Applications (Springer-Verlag, Berlin, 1995).

[19] J. Terry et al., Surf. Sci. 499, L141 (2002).

[20] S. Di Mihai et al., Pulsed Laser Ablation of Solids: Basics, Theory and Applications (Springer-Heidelberg 2014).

[21] R. Eason, Pulsed Laser Deposition of Thin Films: Applications-Led Growth of Functional Materials (John Wiley \& Sons, Hoboken, 2007). 
[22] S. M. Kaczmarek, in SPIE 3187, Szczecin-Swinoujscie, Poland (1997).

[23] T. Venkatesan and S. M. Green, The Industrial Physicist 2, 3 (1996).

[24] E. E. Wisniewski et al., Nucl. Instrum. Meth A 711, 60-64 (2013).

[25] J. P. Perdew et al., Phys. Rev. B 54, 23 (1996).

[26] T. Nakajyo, et al., Jpn. J. Appl. Phys. 42, 1470-1474 (2003).

[27] A. Lorusso et al., Phys. Rev. ST Accel. Beams, 14, 090401-9 (2011).

[28] G. Gatti et al., in Particle Accelerator Conference, Albuquerque, NM, USA (2007).

[29] L. Cultrera et al., Phys. Rev. ST Accel. Beams 12, 043502 (2009).

[30] H. Kaneko et al., Surf. Sci. 602, 2295-2299 (2008).

[31] H. C. Xu, et al., J. Elec. Spec. Relat. Phenom. 200, 347 (2015). 